\title{
Appraisal of ANN and ANFIS for Predicting Vertical Total Electron Content (VTEC) in the Ionosphere for GPS Observations*
}

\author{
${ }^{1}$ Yakubu I., ${ }^{1}$ Ziggah Y.Y. and ${ }^{1}$ Asafo-Adjei D. \\ ${ }^{1}$ University of Mines and Technology, P.O. Box 237, Tarkwa, Ghana
}

Yakubu, I., Ziggah, Y. Y. and Asafo-Adjei. D. (2017), "Appraisal of ANN and ANFIS for Predicting Vertical Total Electron Content (VTEC) in the Ionosphere for GPS Observations", Ghana Mining Journal, Vol. 17, No. 2, pp. $12-16$.

\begin{abstract}
Positional accuracy in the usage of GPS receiver is one of the major challenges in GPS observations. The propagation of the GPS signals are interfered by free electrons which are the massive particles in the ionosphere region and results in delays in the transmission of signals to the Earth. Therefore, the total electron content is a key parameter in mitigating ionospheric effects on GPS receivers. Many researchers have therefore proposed various models and methods for predicting the total electron content along the signal path. This paper focuses on the use of two different models for predicting the Vertical Total Electron Content (VTEC). Artificial Neural Network (ANN) and Adaptive Neuro Fuzzy Inference System (ANFIS) algorithms have been developed for the prediction of VTEC in the ionosphere. The developed ANN and ANFIS model gave Root Mean Square Error (RMSE) of 1.953 and 1.190 respectively. From the results it can be stated that the ANFIS is more suitable tool for the prediction of VTEC.
\end{abstract}

Keywords: Artificial Neural Network, Adaptive Neuro Fuzzy Inference System, Vertical Total Electron

\section{Introduction}

The influence of the ionosphere can reduce the positional accuracy of the GPS receivers especially at the peak of solar flare activities during which the density of the electron content in the ionosphere is high (Kumar and Singh, 2012). It has therefore been recognised that the ionospheric effect is one of the largest sources of errors in GPS navigation. Hence, there is a need to predict VTEC since it aids in the evaluations of the ionospheric effects on radio navigation and communication systems. In line with that, scholars proposed various techniques to help model and correct the ionospheric effect on the GPS signals. Notably among them include but not limited to spherical harmonic functional model, International Reference Ionosphere (IRI) model, Klobuchar model and two-dimensional polynomial ionospheric delay correction model ( $\mathrm{Hu}$ et al., 2014).

In recent times, artificial intelligence algorithms have been proposed and successfully used as alternative to the aforementioned procedures. ANN has been one of the most attractive methods of artificial intelligence for establishing correction models of ionospheric delay. For instance, Habarulena et al., (2007) used backpropagation neural network to establish a VTEC model of a region comprised of various observations stations. $\mathrm{Hu}$ et al., (2014) proposed a hybrid VTEC prediction technique of $\mathrm{ANN}$ and two-dimensional polynomial. In Fan et al., (2010) a generalised regression neural network (GRNN) was utilised to predict the VTEC. Cander et al., (1998) and Sarma and Madhu, (2005) used ANNs to predict the ionospheric layer critical frequency which play a key role in estimating the parameters of the VTEC. Ratnam et al., (2012) predicted the VTEC values over a low latitude GPS stations. In addition to these studies, there are some other ANN based ionospheric delay correction found in the literature (Friedrich et al., 2008; Leandro and Santos, 2007; Ma et al., 2005; Hernandez-Pajares et al., 1997; Tulunay et al., 2006). A general observation made from these ionospheric studies indicate that the ANNs could serve as plausible alternative technique to the classical methods for estimating the VTEC. Despite the widely use of ANNs in VTEC prediction, it has been echoed by several authors that there exist some practical limitations in terms of the network architecture type, the choice of training algorithm and its associated parameters to be used (Fan et al., 2010).

In this study, the adaptive network-based fuzzy inference system (ANFIS) prediction model invented by Jang, (1993) was adopted to predict the VTEC values for the study area. ANFIS is a Sugeno-type fuzzy inference system in which the parameters associated with specific membership functions are computed using either a gradient descent algorithm or in combination with a least squares method. It uses a supervised learning algorithm to optimize parameters of the fuzzy inference system (Akyilmaz and Arslan, 2008). It is worth stating that very few studies have applied the ANFIS technique for VTEC prediction of which better results have been achieved as compared with 
the ANN (Acharya et al., 2011). Therefore, the authors see it as an opportunity to investigate the performance of ANFIS within the area of interest considered in this study. Hence, the strength and extent of applicability (see Table 2 and 3 ) of the ANFIS by making comparison with the ANN for Jiangsu Province, China could be ascertained.

\section{Resources and Methods Used}

\subsection{Resources and Study Area}

Jiangsu Province is located in the middle of the east coast of China. It borders Shandong to the north, Shanghai and Zhejiang to the south and Anhui to the west (Fig. 1). The province is divided into three folds; the south (Sunan), the central (Suzhong) and the north (Subei). It has a coastline of over 1,000 kilometers along the Yellow Sea. Jiangsu is relatively flat, with most of the province not more than 50 meters above sea level. The province has a total surface area of $102,600 \mathrm{~km}^{2}$.It is the densely populated province in China with a total population of 78. 5 million with an average of 771 persons per $\mathrm{km}^{2}$. The population of the province consists of the Manchu, Han and other nationalities. The capital of Jiangsu is Nanjing (Anon., 2017).

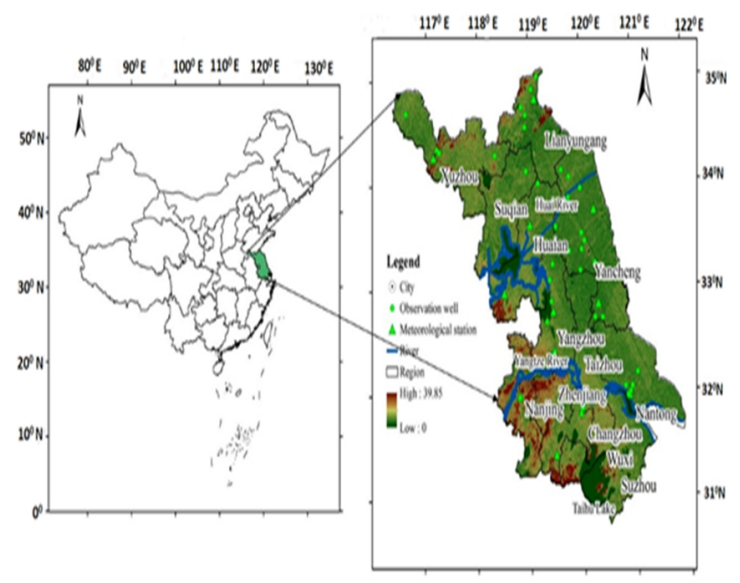

Fig. 1 Map of China showing the location of Jiangsu Provinces (Anon., 2017)

\subsection{Methods Used}

\subsubsection{Artificial neural network (ANN)}

A neural network consists of input layer, hidden layer and output layer as shown in Fig. 2. The input neuron from the input layer represents some independent variable that militate the output result. It is possible to have more than one hidden layer; however, it does not significantly contribute to the accuracy of the output result, but can rather help in the training process (Homan, 2016). Processing of the data take place through the network before they finally arrive at the output layer.
Input Layer Hidden Layer Output Layer

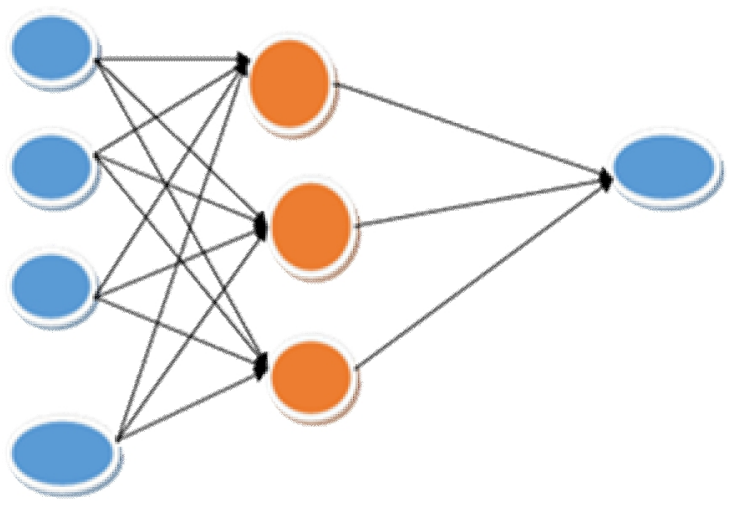

Fig. 2 ANN Structure

However, a network first needs to be trained before interpreting new information. The backpropagation algorithm provides the most efficient learning procedure for multilayer neural networks (Mayilvaganan and Naidu, 2011). In training, the output (predicted) values are compared to the measured (true) data and the error between the two is propagated backwards through the network to update the weights and biases of the network. In this study the Levenberg Marquardt algorithm was used for the weight adaptation and bias. The hyperbolic and linear function were used in the hidden and output layer in the training process. A vector representation of the input and output data is known as the training pairs. The learning process is repeated for all the training pairs until the minimum error of the network is achieved. A total number of 68 data points were used. Table 1 shows a sample of the dataset used in this study. The data set was randomly divided into two parts, $90 \%$ for designing the network and $10 \%$ for testing. Thus, out of the 68 datasets, 61 was used as training and 7 was used to independently assess the optimum trained model. In the ANN and ANFIS training, the latitude and longitude were used as the input data and the observed VTEC was used in the output layer. This conforms to the supervised learning algorithm that was utilized in this study. The training and testing root mean square error (RMSE) values were monitored and the architecture that produced the least RMSE for the test data was selected as the optimum ANN model. The RMSE was estimated using Equation 1.

$$
R M S E=\sqrt{\frac{1}{N} \sum_{i=1}^{N}(y-\bar{y})^{2}}
$$

where $\mathrm{N}$ is the number of data points, $\mathrm{y}$ is the VTEC predicted values and $\bar{y}$ is the VTEC measured values from the GPS receivers. 
Table 1 Sample of data used for the ANN and ANFIS formulation

\begin{tabular}{|c|c|c|c|}
\hline $\begin{array}{c}\text { Station } \\
\text { number }\end{array}$ & IPP lat $^{\mathbf{0}}$ & IPP long $\boldsymbol{}^{\mathbf{0}}$ & $\begin{array}{c}\text { Observed/ } \\
\text { TECU }\end{array}$ \\
\hline 13 & 33.553 & 115.766 & 11.172 \\
\hline 9 & 33.756 & 109.973 & 10.315 \\
\hline 66 & 33.262 & 115.781 & 9.057 \\
\hline 20 & 32.959 & 113.458 & 11.667 \\
\hline 57 & 31.202 & 110.45 & 13.461 \\
\hline 10 & 32.191 & 115.787 & 11.544 \\
\hline 12 & 36.01 & 112.112 & 11.172 \\
\hline
\end{tabular}

\subsubsection{Adaptive Neuro Fuzzy Inference System} (ANFIS)

ANFIS is a three-layer feed forward network: the first layer represents input variables, the second layer is a hidden layer which represents the fuzzy rules, and the last layer represent the output. ANFIS uses hybrid training algorithm based on the combination of least squares estimation and gradient descent algorithm (Akyilmaz and Kutterer, 2004). The shape of the membership functions is defined based on the parameters obtained as a result of the backpropagation algorithm (Mayilvaganan and Naidu, 2011). These parameters are known as the premise parameters. However, in the hybrid training algorithm, the consequent parameters are derived as a result of the least squares estimate. The error obtained is propagated backward and the premise parameters are updated by the gradient descent algorithm (Jang, 1993). It must be known that the same number of training and testing data sets used in the ANN model was applied in the ANFIS model development. The ANFIS structure formed is shown in Fig. 3.

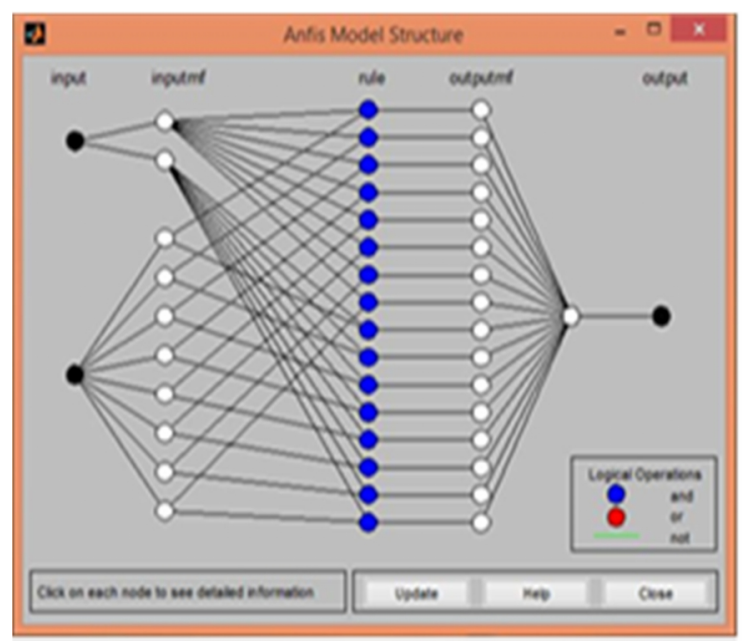

Fig. 3 ANFIS Structure for VTEC Model

\section{Results and Discussion}

\subsection{Results}

The test results for the ANN and ANFIS were compared to determine the technique with higher degree of prediction accuracy. A number of 10 neural networks gave the best result for the ANN. With the various ANFIS configurations performed, 28 membership functions with 250 epochs gave the best result or an acceptable number of parameters with the smallest RMSE. The number of linear parameters and nonlinear parameters were 48 and 20 respectively. The total number of nodes for a given training data set was found to be 57 . Table 2 shows the results produced by the ANN and ANFIS models.

Table 2 Predicted Test Result of the ANN and ANFIS (units: TECU)

\begin{tabular}{|c|c|c|c|}
\hline $\begin{array}{c}\text { Station } \\
\text { number }\end{array}$ & Observed & ANN & ANFIS \\
\hline 13 & 11.172 & 11.710 & 10.000 \\
\hline 9 & 10.315 & 11.751 & 9.480 \\
\hline 66 & 9.057 & 12.114 & 10.100 \\
\hline 20 & 11.667 & 10.810 & 11.400 \\
\hline 57 & 13.461 & 9.900 & 11.300 \\
\hline 10 & 11.544 & 10.405 & 10.200 \\
\hline 12 & 11.172 & 11.159 & 11.600 \\
\hline
\end{tabular}

Statistical analysis was performed on the residuals generated by the models to determine the best model for VTEC prediction in the study area. The criterion used to assess the goodness of fit for the ANN and ANFIS models was based on the RMSE and relative error (Equation 2).

$$
E_{r}=\frac{1}{N} \sum_{i=1}^{N} \frac{|y-p|}{y} \times 100
$$

Here, $\mathrm{y}$ is the observed VTEC, $\mathrm{p}$ is the predicted VTEC and $\mathrm{N}$ is the number of observation. A Summary statistics on the performance of ANN and ANFIS is shown in Table 3.

Table 3 Performance Assessment of the ANN and ANFIS Models

\begin{tabular}{|l|l|l|}
\hline $\begin{array}{c}\text { Model } \\
\text { Type }\end{array}$ & RMSE/TECU & $\begin{array}{c}\text { Relative } \\
\text { Error/TECU }\end{array}$ \\
\hline ANN & 1.953 & 14.426 \\
\hline ANFIS & 1.190 & 9.131 \\
\hline
\end{tabular}




\subsection{Discussion}

The ANN produced RMSE of 1.953 while 1.190 was given by ANFIS. The obtained RMSE test results indicate the extent of agreement between the observed data points and the models' predicted VTEC values. Thus, the model with the least RMSE value had a better fit to the observed data. On the basis of that, it can be stated that the ANFIS outperformed the ANN. Therefore, the inference made here is that the ANFIS technique has demonstrated a better calibration ability and good generalisation performance across the entire dataset than the ANN method. In addition to that, the ANFIS being a hybrid model uses both the strengths and weaknesses of ANN and Fuzzy logic to complement each other. Thus, the approach is able to combine the ANN and Fuzzy logic function estimation and nonlinear modelling capabilities. The superiority of the ANFIS to ANN was further demonstrated when comparison was made between their obtained relative error value (Table 3 ). In Table 3, the ANFIS had 9.131 TECU while the ANN produced 14.426. This means that the ANFIS can predict accurately to about $90.69 \%$ of the observed VTEC value from the GPS while ANN could predict up to about $85.57 \%$. This can additionally be viewed from Figs. 4 and 5 where the degree of variation of the predicted and observed as well as that of the residuals can clearly be seen for both ANFIS and ANN respectively.

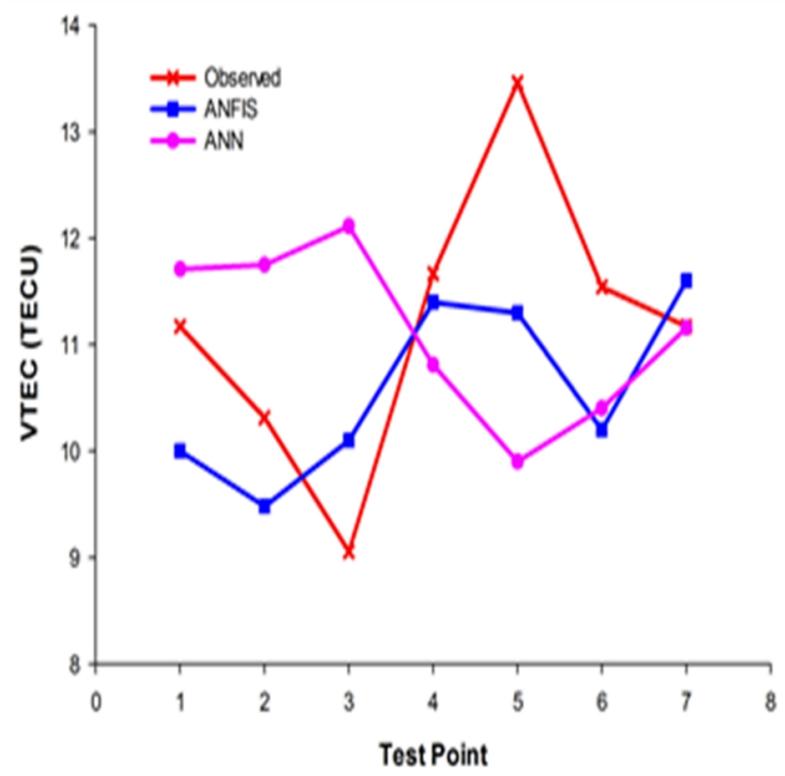

Fig. 4 ANN and ANFIS predicted VTEC

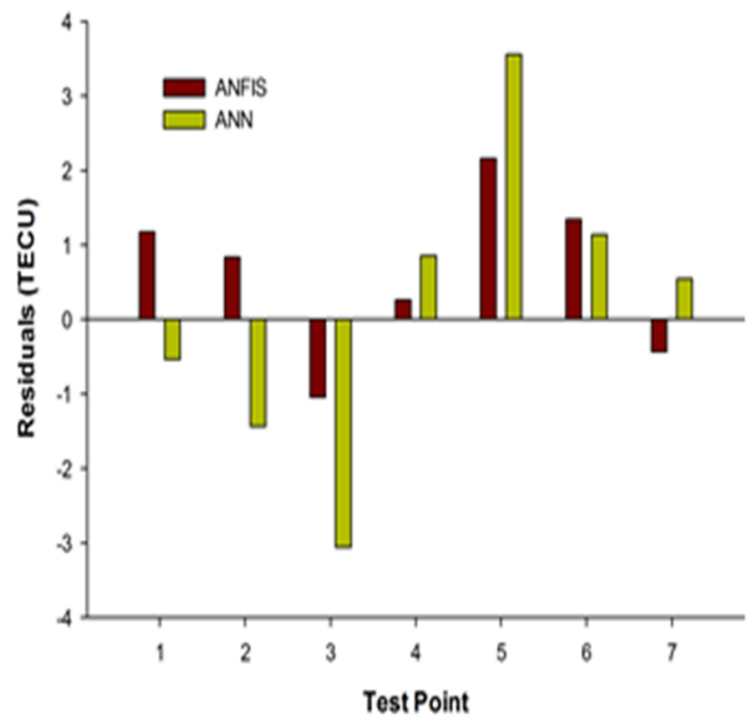

Fig. 5 ANN and ANFIS predicted VTEC

\section{Conclusions}

The ionosphere is one of the atmospheric layers that causes the delay in the signal propagation from the satellite to the receiver. This ionospheric delay has been regarded as a major source of error that influences the performance of GPS receivers. Scholars have therefore applied several methods to correct such defect. In this study, a comparison of two computational intelligent algorithms namely, ANN and ANFIS have been presented. The ANFIS-derived results with that of ANN have been analysed based on the RMSE and relative error. The overall statistical analyses indicate that the ANFIS is a suitable tool for the prediction of VTEC in short-term range. Moreover, the ANFIS is computationally efficient as it takes shorter time to complete training compared to the ANN. The study has also revealed that the ANFIS does not require a large number of training dataset for an optimal prediction, whereas a considerable number of training dataset have to be considered during ANN training.

\section{References}

Acharya, R., Roy, B., Sivaraman, M. R. and Dasgupta, A. (2011) "Prediction of Ionospheric Total Electron Content using Adaptive Neural Network with in-situ Learning Algorithm", Advances in Space Research, Vol. 47, No. 1, pp. 115-123.

Akyilmaz, O. and Kutterer, H. (2004), "Prediction of Earth Rotation Parameters by Fuzzy Inference Systems", Journal of Geodesy, No. 78, pp. 82-92.

Anon., (2017), "China Cities and Provinces", www.chinatoday.com/city/jiangsu.htm.

Accessed: March 15, 2017. 
Cander, Lj. R., Milosavljevic, M. M., Stankovic, S. S. and Tomasevic, S. (1998), "Ionospheric Forecasting Technique by Artificial Neural Network", Electronics Lett., Vol. 34, Issue 16, pp. 1573-1574.

Fan, G., Wang, W. and Xi, X. (2010), "Modeling of Ionosphere VTEC using Generalized Regression Neural Network" Acta Geodaetica et Cartographica Sinica, Vol. 39, No. 1, pp. 16-21.

Friedrich, M., Fankhauser, M., Oyeyemi, E. and McKinnell, L.A. (2008), "A Neural NetworkBased Ionospheric Model for Arecibo", Advances in Space Research, Vol. 42, No. 4, pp. 776-781.

Habarulema, J. B., McKinnell, L. A. and Cilliers, P. J. (2007), "Prediction of Global Positioning System Total Electron Content using Neural Networks Over South Africa" Journal of Atmospheric and Solar-Terrestrial Physics, Vol. 69, No. 15, pp. 1842-1850.

Hernandez-Pajares, M., Juan, J. M. and Sanz, J., (1997), "Neural Network Modeling of the Ionospheric Electron Content at Global Scale using GPS Data", Radio Science, Vol. 32, No. 3, pp. 1081-1089.

Homam, M. J. (2016), "Prediction of Total Electron Content of the Ionosphere Using Neural Network", Jurnal Teknologi, Vol. 78, pp. 53-57.

Hu, W. S., Zheng, D. Y. and Nie, W. F (2014), "Research on Methods of Regional Ionospheric Delay Correction based on Neural Network Technology", Survey Review, Vol. 46, pp. 1-8.

Jang, J. R. (1993), "Adaptive Network Based Fuzzy Inference System”, IEEE Transactions on System Man and Cybernetics, Vol. 23, No. 3, pp. 665-685.

Kumar, S. and Singh, A. K. (2012), "Effect of Solar Flares on Ionospheric TEC at Varanasi, Near EIA Crest, During Solar Minimum Period", Indian Journal of Radio and Space Physics, Vol. 41, pp. 141-147.

Leandro, R. F. and Santos, M. C. (2007), "A Neural Network Approach for Regional Vertical Total Electron Content Modelling”, In Stud. Geophys. Geod., Vol. 51, pp. 279-292.

Liu, L. Huang, L., Chen, J., Wu, P. and Qin, X. (2017), "Applicability Analysis of VTEC Derived from Sophisticated Klobuchar Model in China", ISPRS International Journal of GeoInformation, Vol. 6, No. 75, pp. 1-15.

Ma, X. F., Maruyama, T., Ma, G. and Takeda, T. (2005), "Three-Dimensional Ionospheric Tomography using Observation data of GPS Ground Receivers and Ionosonde by Neural Network", Journal of Geophysical Research: Space Physics, Vol.110, pp. 2156-2202.

Mayilvaganan, M. K. and Naidu, K. B. (2011), "Comparative Study of ANN and ANFIS for the Prediction of Groundwater Level of a Watershed", Global Journal of Mathematical
Science: Theory and Practical, Vol. 3, No. 4, pp. 299-306.

Ratnam, D. V., Dinesh, B. V., Tejaswi, B., Kumar, D. P., Ritesh, T. V., Brahmanadam, P. S., and Vindhya, G. (2012), “TEC Prediction Model using Neural Networks over a Low Latitude GPS Station”, International Journal of Soft Computing and Engineering (IJSCE), Vol. 2, Issue 2, pp. 517-521.

Sarma, A. D. and Madhu, T., "Modelling of foF2 Using Neural Networks at an Equatorial Anomaly Station", Current Science, Vol. 89, No. 7, pp. 1245-1247.Tulunay, E., Senalp, E. T., Radicella, S. M., and Tulunay, Y. (2006), "Forecasting Total Electron Content Maps by Neural Network Technique", Radio Sci., Vol. 41, No. 4, pp. 4- 16.

\section{Authors}

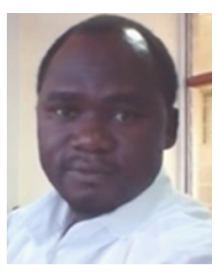

Dr Issaka Yakubu is a Senior Lecturer at the Geomatic Engineering Department of the University of Mines and Technology (UMaT), Tarkwa-Ghana. He holds a Certificate in Disaster Risk Management and Environmental Assessment for Spatial Planning from the International Institute for Geo-information Science and Earth Observation (ITC)-University of Twente, The Netherlands and BSc. degree in Geomatic Engineering from the Kwame Nkrumah University of Science and Technology, Kumasi-Ghana. He obtained his Master of Philosophy degree and Doctor of Philosophy from UMaT. His research interests include Global Navigation Satellite System (GNSS) and Applications, Geo-Information for Disaster Management, GeoInformation for Fleet Management and Land Policy and Administration.

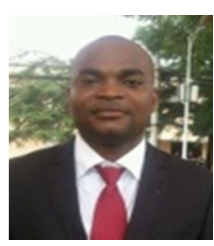

Dr Yao Yevenyo Ziggah is a Lecturer at the Geomatic Engineering Department of the University of Mines and Technology (UMaT). He holds a BSc in Geomatic Engineering from Kwame Nkrumah University of Science and Technology, Kumasi-Ghana, He obtained his MEng degree and Doctor of Philosophy in Geodesy and Survey Engineering from China University of Geosciences (Wuhan). His research interests include geodetic deformation modelling, geoid modelling, geodetic coordinate transformation and application and development of Artificial Intelligence Techniques in Geodesy.

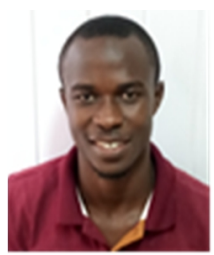

Mr Asafo, Adjie. David holds BSc Degree in the Geomatic Engineering Department from the University of Mines and Technology (UMaT). He is currently a National Service Personnel at UMaT. His research interest includes GNSS and Applications, Geodesy and Engineering Surveying 